\title{
LE SOCIALISME ET LE MOUVEMENT OUVRIER A BARCELONE A LA CHUTE D'ISABELLE II $^{1}$
}

\section{INTRODUCTION}

Dans cet article nous nous proposons de dévoiler, autant que possible, les origines souvent très cachées des opinions différentes et divergentes qui après la révolution de septembre 1868 , lorsque le mouvement ouvrier pouvait s'exprimer librement, s'y sont manifestées. A cet effet nous devrons décrire le milieu où le mouvement s'est développé pendant les années qui précèdent immédiatement la révolution, aussi bien pendant les périodes où on ne pouvait travailler que dans la clandestinité qu'aux moments où le gouvernement avait une attitude tolérante vis-à-vis du mouvement. Or, il est indispensable de décrire de façon globale quelques-unes des forces les plus importantes qui ont déclanché la révolution en question. ${ }^{2}$

\section{LE PARTI PROGRESSISTE}

L'attitude adoptée par le parti progressiste aux élections d'octobre $186_{3}$, et qui consistait à se retirer, attitude prise également aux élections suivantes de I 864 et I 865 , fut un acte de révolte évidente et publique qui, à la fin, devait déclancher la révolution de septembre i 868 . Le parti imita, avec cette attitude, qui annonce pratiquement la révolution,

1 Cet article est un résumé d'un chapitre de l'ouvrage de l'auteur, Orígenes del Anarquismo en Barcelona, qui fut présenté comme thèse doctorale à la Faculté des Sciences Sociales de l'Université Grégorienne de Rome, le mois janvier 1958, et qui paraîtra au cours de l'année 1959 à Barcelone, Editorial Teide. Une seconde partie de cet ouvrage sera publiée dans un numéro prochain de ce périodique.

2 Le déclin progressif du régime monarchique sous Isabelle forme le sujet d'une grande étude de R. Olivar Bertrand, Así cayó Isabel II, Barcelone I955. La bibliographic se trouve aux pp. 4I 5-417. Les lettres des hommes politiques progressistes que l'auteur a pu utiliser et qu'il a publiées à la fin de son ouvrage, sont d'un grand intérêt. Cf., en outre, la riche bibliographie sur cette époque présentée par A. Ballesteros, Historia de España y de su influencia en la Historia Universal, T. VIII, Barcelone 1936, pp. $9^{8}$ ss. 
celle du dernier des Horaces: „faisant semblant de se retirer, il assurait le succès de la lutte". ${ }^{1}$ Dans la langue journaliste de l'époque on tâchait ainsi de donner un ton de dignité classique à ce geste de dépit des politiciens progressistes. Avides de pouvoir ils ne supportèrent pas la circulaire du ministre Vaamonde qui, comme chacun aurait pu le prévoir, commençait à réintroduire l'influence morale si connue de l'équipe au pouvoir sur les élections. ${ }^{2}$

La dynastie ne sut pas se placer au dessus des maniements des partis. Après l'échec de i 863 de l'Union Libérale, dirigée par O'Donnell, qui s'était maintenu au pouvoir pendant cinq ans, la reine Isabelle se passa encore des progressistes lors de la constitution de nouveaux gouvernements. Le manifeste du parti progressiste publié le 29 octobre I864, afin de faire connaitre les raisons de sa deuxième retraite, signale à quatre reprises les célèbres obstacles traditionnels, expression employée par Olózaga aux Sessions du Congrès de i I et de 12 décembre I 86I, quand il parlait des camarillas et de l'influence des courtisans. ${ }^{3}$

Il ne faut pas s'étonner de ce que le parti progressiste, porté toujours pour la monarchie constitutionnelle, voyant la reine se livrer inconditionnellement aux mains des modérés, s'approcha des positions du parti démocratique, antimonarchiste au fond, afin de s'opposer aux attaques de la réaction.

Les progressistes ne se proposaient de combattre que la dynastie, tandis que les démocrates s'apprêtaient à combattre la monarchie elle-même. Le pacte explicite entre les deux partis fut scellé d'abord à Ostende, le I 6 août I 866 , après les essais révolutionnaires du Général Prim pendant les mois de janvier et juin de cette même année, qui échouèrent - et puis, à Bruxelles, le 30 juin 1867 , avant l'insurrection du mois d'août de la même année, qui n'eut également pas de résultat. La clause, qui soumettait vaguement à la décision d'une Assemblée constituante, élue par suffrage universel, la question constitutionnelle, d'opter pour la monarchie ou pour la république, permit aux deux partis d'adopter ces communes résolutions, sans porter préjudice à leurs principes respectifs. ${ }^{4}$

1 L'expression figure dans un article du journal progressiste de Madrid, La Iberia, où l'on tente de justifier la décision du parti progressiste de ne pas participer aux élections de I863. Voir cet article dans le journal progressiste de Barcelone, La Corona, i I septembre I863, no. $4^{10}$, P. I.

2 Olivar Bertrand, o.c., pp. 83, 102, II $3,146-I_{47}$ et 169 , fait mention des manipulations électorales auxquelles le gouvernement a recours à plusieurs reprises.

3 Ibid., p. 107, n. 29.

4 Ibid., pp. I56 et 172 . Cf., en outre, son ouvrage El caballero Prim, v. II, Barcelone I952, Pp. 2 I I et 223. 


\section{LE PARTI DÉMOCRATIQUE}

S'il est vrai qu'on doit attribuer au parti progressiste en général, et plus spécialement à Prim, la direction effective des forces qui déclanchèrent la chute d'Isabelle II et consacrèrent l'entrée des milieux populaires sur la scène politique moyennant le suffrage universel ${ }^{1}$, le parti démocratique nous intéresse dans cette étude, parce qu'il se trouvait le plus près de ce secteur de la classe ouvrière qui fit son apparition dans la vie publique de l'époque avec les manifestations d'un vrai mouvement: presse, congrès, programme d'aspirations de classe, etc.

Le fait qu'il y avait plusieurs courants dans le parti démocratique était devenu évident depuis la dispute de I 860 entre J. M. Orense, Marquis d'Albaida, et Fernando Garrido, au sujet d'une attaque personnelle du premier contre celui-ci à propos du socialisme, ${ }^{2}$ ce qui provoqua la soi-disant „Declaración de los Treinta”. Cette déclaration des premiers hommes du parti démocratique laissait la possibilité aux membres du parti de s'attacher à n'importe quels principes sociaux ou économiques, et restreignait les dogmes du parti au domaine exclusivement politique. ${ }^{3}$

Les oppositions qu'il y avait entre ces divers courants dans la démocratie acquirent une acuité particulière au printemps de 1864 , lorsque Pi y Margall, devenu le directeur de La Discusión depuis le ier avril de cette même année, publia dans cette revue son article „La

${ }^{1}$ La révolution de septembre 1868 a été réalisée sous la devise: „Viva la soberanía nacional !” Cf. les bans des différentes ,juntas revolucionarias” rassemblés par D. M. M. Lara, E1 Cronista de la Revolución española de I 868, Barcelone I 869. Les problèmes juridiques posés par la „constitutionnalisation" progressive de la monarchie absolue en France et en Espagne ont été différents, d'accord avec la tradition du droit politique dans chacun de ces deux pays. Cf. sur ce sujet Luis Diez del Corral, El liberalismo doctrinario, Madrid 1945, pp. $62-68$ et $460-463$.

2 Cf. Eugenio García Ruiz, La democracia, el socialismo y el comunismo, según la filosofia y la historia, Madrid I 861; et Francisco Caravaca, Pi y Margall, Barcelone 1935, pp. 58 et 59. Cf. aussi le journal démocratique de Madrid, La Discusión, 26 mai I 864, no. 2585, p. 3 .

3 Cette déclaration était: „Los que suscriben declaran que consideran como demócratas indistintamente a todos aquellos que, cualesquiera que sean sus opiniones en filosofía y en cuestiones económicas y sociales, profesen en política el principio de la personalidad humana, o de las libertades individuales, absolutas e ilegislables, y del sufragio universal, así como los demás principios politicos fundamentales consignados en el programa democrático". Cf. Eugenio García Ruiz, o.c., p. r9x.

Ce débat et la déclaration conclusive ont donné lieu à quatre publications sur le socialisme. Partent d'un point de vue strictement libéral, E. Garcia Ruiz, o.c.; Ceferino Tresserra, $¿$ Los anarquistas, los socialistas y los comunistas, son demócratas?, Barcelone $186 \mathrm{I}$; et Adolfo Joarizti, Los progresistas, los demócratas y los individualistas, Madrid I86I, et d'un point de vue socialiste, Fernando Garrido, El socialismo y la democracia ante sus adversarios, Londres 1862 . 
propiedad", 1 qui marqua le commencement de la célèbre polémique entre Castelar et lui.

\section{LE COURANT SOCIALISTE}

L'orientation de Pi y Margall est déjà suffisamment mise en évidence dans son livre La reacción y la revolución, publié en 1854 . On lit dans sa première page: „Notre révolution n'est pas purement politique, elle est sociale." 2 D'après lui, la réforme à laquelle la société devait être soumise, ne devait pas se borner au seul aspect ,formel" et extrinsèque ${ }^{3}$ d'un changement du système gouvernemental ou d'une certaine amplification du suffrage, mais elle devait toucher la base même de la société: l'économie. ${ }^{4}$

La place éminente qui devait être occupée par le problème économique dans les buts du parti démocratique est signalée dans des termes pas trop directes dans la déclaration des intentions publiée par la nouvelle rédaction de La Discusión dans le numéro d'avril $\left.18644^{5} \mathrm{I}\right]$ y a deux sortes de raisons, d'après Pi y Margall, pour lesquelles le

1 La Discusión, 22 avril 1864 , no. 2557, p. 1 .

${ }^{2}$ F. Pi y Margall, La reacción y la revolución, Madrid i 854 , p. 3. Il y a une autre édition à Barcelone, I928, par La Revista Blanca.

${ }^{3} \mathrm{Pi}$ y Margall n'emploie pas encore ces épithètes dans l'ouvrage cité qui date de 1854. Ultérieurement, au cours de la polémique avec Castelar, il écrira dans son article: ¿Somos socialistas?, dans La Discusión, 17 mai I 864 , no. 2577, p. I: „Las [réformes] políticas apenas afectan más que la vida exterior de los pueblos; las sociales afectan su vida íntima." "F. Pi y Margall, La reacción y la revolución, o.c., p. 204, écrit: „[...] para fundarla [la nouvelle société] no sólo es necesario acabar con la actual organización política, sino también con la económica; [...] es indispensable, no ya reformar la nación, sino cambiar la base." Et à la p. 229: „La revolución es hoy tan social como política. Se propone reformar las naciones no sólo en su organismo, sino también en lo que las constituye esencialmente. He dicho ya que tiende a la destrucción del poder [cf. pp. 202 et 203], a la celebración de un contrato. Todo contrato es un acto de justicia conmutativa; la justicia conmutativa, del dominio de la economía. La revolución se compromete, por lo tanto, a armonizar las fuerzas económicas, o lo que equivale a lo mismo, a resolver el oscurísimo problema [du travail et du capital]." Dans ce livre on ne parle qu'accidentellement de l'économie, parce que, comme dit l'auteur (pp. 94, I93, 229 et 349), les questions spécifiquement économiques auraient dû être traitées dans un troisième volume qui jamais ne fut publié.

Cette manière de voir le problème social et le problème politique coincide, pour l'essentiel, avec celle des socialistes italiens Ferrari et Pisacane, contemporains de Pi y Margall. Leur pensée et l'influence de Proudhon sur eux ont été étudiées par Franco della Peruta, Il socialismo risorgimentale di Ferrari, Pisacane e Montanelli, dans Movimento Operaio (Milano) [1956], pp. 3-4I.

5 „[La démocratie] ha de llevar su pensamiento y su mano no sólo a la constitución del poder, sino también a todos y cada uno de los ramos de la administración pública; no sólo a la administración, sino también a las leyes criminales y civiles y al orden de relaciones que establece entre los hombres el cambio de servicios y productos. Los probiemas de que antes se ha hablado versan principalmente sobre este orden de relaciones." De l'article Nuestra conducta política, dans La Discusión, I avril $x 864$, no. 2538 , p. I. 
champ économique est un élément essentiel de la révolution poussée par le parti démocratique: des raisons historiques et des raisons philosophiques.

Historiquement, dans les luttes politiques „les classes victorieuses éprouvent le besoin de désarmer les vaincus, leur enlevant la base du pouvoir: la richesse". ${ }^{1}$ Se référant aux révolutions du XIX sièclelui-même, Pi y Margall affirme que la classe bourgeoise (jusqu'en i 868 appelée infailliblement „classe moyenne"), à la suite des réformes économiques auxquelles elle a soumis la noblesse et le clergé, a entrepris sa „révolution sociale", d'ailleurs à son propre profit. La révolution poussée par le parti démocratique tient tout simplement à augmenter le nombre des bénéficiaires, à émanciper les classes salariées autant au point de vue politique que social, et à leur faire place ,au banquet de la vie" ${ }^{2}$, ce qui a des répercussions économiques indubitables.

Dans le domaine de la philosophie une des idées fondamentales de Pi y Margall est celle de l'autonomie. Cette idée résume, d'une part, son attitude profondément rationaliste vis-à-vis de l'ensemble des problèmes humains: l'être humain se suffit ${ }^{3}$ et il est libre de toute soumission à toute sphère transcendante. D'un autre côté, dans cette conception rationaliste de l'existence humaine, l'autonomie de l'homme se dédouble: l'autonomie de l'individu existe ainsi que celle de la collectivité; chacune d'elles avec son propre espace inviolable.

Le domaine de l'autonomie individuelle comprend ,les actes de la vie individuelle, [...] les manifestations de la pensée et de la conscience, [...] la manifestation de l'être intérieur". ${ }^{4}$ Chaque personne humaine, voilà le sujet de ces droits à la vie et de cette liberté de conscience, de pensée et d'expression. Ces droits personnels sont absolus, inaliénables et inviolables par quelque puissance humaine que ce soit.

„Les actes de la vie sociale, c'est à dire, [...] tous les actes qui concernent la propriété, l'échange, l'organisation du pouvoir et l'administration des intérêts généraux" 5 , voilà le domaine de l'autonomie collective. La collectivité, considérée par Pi y Margall non comme un simple être moral, mais comme un être authentique et réel, „qui pense,

1 F. Pi y Margall, La revolución actual y la revolución democrática, dans La Discusión, I avril I 864 , no. 2538 , p. I.

2 F. Pi y Margall, ibid., p. 2.

${ }^{3}$ F. Pi y Margall, Principios, dans La Discusión, 8 avril 1864 , no. 2545, p. I. Cf. aussi les affirmations positives de La reacción y la revolución: „La ciencia moderna ha reconocido en nosotros [...] que somos la fuente de toda certidumbre y de todo derecho" (p. 200). „Homo sibi Deus, ha dicho un filósofo alemán: el hombre es para sí su realidad, su derecho, su mundo, su fin, su Dios, su todo [...]" (p. 202).

4 F. Pi y Margall, Las libertades económicas, dans La Discusión, I 3 avril I 864, no. 2549 , p. I.

$\mathbf{5}$ Ibidem. 
qui sent et qui agit" ${ }^{1}$, doit avoir la compétence d'agir dans ce domaine. Contre la tendance strictement libérale qui conçoit l'économie comme dominée par des lois fatales tout à fait au delà de n'importe quelle ingérence juridique positive - tendance qui consiste à ériger, sur le terrain économique, le „sauve qui peut" pratiquement comme principe de gouvernement ${ }^{2}$ - Pi y Margall proclame la légitimité de l'intervention de l'Etat „par des institutions et des lois" 3, parce que, d'après lui, l'Etat est le dépositaire et le sujet des droits collectifs, sans pouvoir en exclure les autres collectivités. ${ }^{4}$

En conséquence, une révolution exclusivement politique devient

1 Ibidem. - Il n'y a pas de doute que sur ce point depuis l'époque de La reacción y la revolución, 1 854, jusqu’à 1 864, la pensée de Pi y Margall évolue considérablement ce que nous ne ferons ici que signaler. D'après sa propre confession, c'est la découverte que les êtres collectifs existent réellement qui a déclanché cette évolution. [Cf. F. Pi y Margall, Las cartas del Sr. Rivero, dans La Discusión, 26 mai 1864, no. 2585.] - La reacción y la revolución est dominée par l'idée proudhonienne de la souveraineté de l'individu, absolument ingouvernable; tout pouvoir est absurde (pp. 202-204). Cette orientation était encore celle de Pi y Margall en 1857 (cf. la deuxième lettre de Nicolás M. Rivero dans La Discusión du 26 mai mentionnée, p. 2, ainsi que les reproches de La Democracia du i I et 14 juin 1864 , nums. 136 et 138 , pp. 2 et 1 respectivement. - Une étude complète de cette affaire devra tenir compte des articles écrits par Pi dans La Discusión de 1857, mentionnés par E. Vera González: Pi y Margall y la política contemporánea, Barcelone, Vol. I, I886, pp. 593-594). Vera y González, tout en étudiant en détail le livre La reacción y la revolución (pp. 473-500) et qui reproduit textuellement l'article mentionné Las cartas del Sr. Rivero, ne parle même pas de ce changement subi par Pi y Margall. - Bien que dans le livre La reacción y la revolución, il affirme déjà qu'il y a identité entre l'être et l'idée et il formule le principe que ce qui est le général constitue l'essentiel de chaque chose - ce qui l'amène jusqu'à une valorisation très poussée de l'espèce comme telle - son attitude est pourtant hésitante: il s'aperçoit du danger que cette valorisation de l'espèce peut représenter pour la souveraineté de l'individu, et il ne cache pas qu'il hésite entre les deux thèses qui sont antinomiques dans un certain sens. En somme, bien que l'idée que les sociétés sont des entités indépendantes, distinctes des individus s'exprime déjà en principe dans le livre La reacción y la revolución de 1894 , il faut aussi avoucr que dans la conscience de Pi y Margall, à cette époque-là, les thèses proudhoniennes de la souveraineté de l'individu avaient un poids décisif. Voilà la raison de ses doutes et de ses hésitations théoriques, manifestées dans le chapitre IX de l'ouvrage mentionné, et plus spécialement aux pp. 244-247. - A propos du rôle assigné à l'espèce humaine d'après les idées que l'auteur a sur le progrès et la révolution, cf. tout le chapitre II, spécialement les pp. 88-91; en plus les pp. 66 et 68 .

${ }^{2}$ F. Pi y Margall, ¿ Somos socialistas?, dans La Discusión, 17 mai I 864, no. 2577, p. I. ${ }^{3}$ Cf. l'entrefilet polémique contre le journal de Castelar La Democracia, dans La Discusión, 22 mai 1864 , no. 2582 , p. 2.

" Ibidem. Il écrit par exemple: „[...] la libertad económica debe estar regularizada y subordinada al interés colectivo. El Estado debe ser, no sólo la garantia, sino la realización del derecho. Mientras el derecho no sea una realidad, ¿cómo ha de garantirlo el Estado? Y el derecho, ¿ cómo se realiza en las sociedades, sino por instituciones y leyes?”. - L'État, dans l'ordre concret, réalise la société "substantive"; cf. l'entrefilet de La Discusión, 26 mai I 864, no. 2585 , p. 3: „Nosotros derivamos la idea que del Estado tenemos de la realidad y substantividad de las sociedades," 
tout à fait inimaginable: l'Etat ne peut pas se désintéresser des problèmes économiques qui constituent, en grande partie, „la sphère d'activité de l'autonomie des sociétés". 1

Néanmoins, le domaine de l'activité de l'Etat reste théoriquement borné par l'autonomie de l'individu, aussi consistente et aussi valable que celle de la collectivité. ${ }^{2}$ Pi y Margall se déclare ennemi d',une sorte de panthéisme social et politique" dans lequel l'Etat absorbe complètement l'individu. ${ }^{3}$ En plus de son indépendence générique dans ce domaine de son autonomie individuelle, il reconnaît le droit de propriété de l'individu aux fruits de son travail, et, quant à la propriété de terres, soutenant même qu'elle se trouve soumise à la collectivité, qui ,a le droit de la légiférer, de la reformer et de l'adapter à la manière de sentir et d'être, aux successives nécessités de la vie", 4 il affirme en même temps que la terre doit être ,en possession de l'individu". 5

En résumant, le socialisme de Pi y Margall pourrait être caractérisé d'après son principe fondamental de l'entité indépendante des êtres collectifs. ${ }^{6} \mathrm{Ce}$ "réalisme social” établit la base des droits de la collectivité, voire de l'Etat, sur l'économie. L'autonomie de la collectivité - de l'Etat - dans sa propre sphère qui enferme les relations économiques, introduit le droit, l'ordre juridique dans le domaine de l'économie ${ }^{7}$ et y consacre définitivement l'intervention de l'Etat. Voilà les idées socialistes que Pi y Margall exposa dans La Discusión, en polémique contre Castelar. Comme nous aurons encore l'occasion de constater par la suite, la polémique sur le socialisme entre Pi y Margall et Castelar ne se borna pas à exprimer la différence d'estimations personnelles entre ces deux hommes politiques, mais ce fut plutôt un

1 Selon l'article A La Democracia, dans La Discusión, 25 mai 1864, no. 2584, p. I., y sont comprises: "1 ${ }^{\circ}$, la constitución del poder público; $2^{\circ}$, la regularización de las fuerzas económicas; o sea, la determinación de las relaciones creadas por la propiedad, el trabajo y el cambio."

2 „Ambas igualmente legítimas, ambas igualmente necesarias”, écrit-il de l'autonomie individuelle et de l'autonomie collective. F. Pi y Margall, Las libertades económicas, dans La Discusión, 13 avril I 864, no. 2549, p. 1.

${ }^{3}$ F. Pi y Margall, ¿Somos socialistas?, dans La Discusión, $x 7$ mai 1864 , no. 2577 , p. $x$.

4. Pi y Margall, La propiedad, dans La Discusión, 22 avril 1864 , no. 2557, P. I.

5 Ibidem.

- L'importance de ce principe apparaît dans tout ce que Pi y Margall a écrit dans le journal La Discusión pendant l'annéc r 864 . Dans un entrefilet du 20 mai I 864 , no. 2580 , p. $x$, on peut lire: „Por socialismo, en su acepción lata, se entiende la teoría que, teniendo por objeto la organización social, se funda en el reconocimiento previo de la sociedad como ser real, sustantivo y orgánico con todos los individuos que lo constituyen."

7 Cf. l'article de F. Pi y Margall, ¿'Somos socialistas?, dans La Discusión, 17 mai 1864, no. 2577, p. I. Et, par sa remarquable fidélité à l'esprit de Pi y Margall, celui de J. Rodríguez y Morales, ¿Qué es la reacción? ¿Qué es la revolución?, dans La Discusión, 7 mai 1864, no, 2569 , p. I, 
nouveau témoignage, plus convainquant si on veut, des différences profondes qu'il y avait entre les deux courants qui existaient dans le parti démocratique depuis quelques années. ${ }^{1}$

\section{LE COURANT INDIVIDUALISTE}

Emilio Castelar et ceux qui avaient une opinion contraire à celle de Pi y Margall prennent déjà dans les colonnes de La Democracia comme point de départ les prémisses de l'école liberale. La valeur suprême de l'homme c'est la liberté. Les lois qui régissent la société humaine „ne sont dans leur base et dans leur forme, que les lois mêmes de la nature. La loi caractéristique de la nature [...] c'est la liberté".2

L'Etat, simple tuteur de l'ordre et de la liberté, viole les limites de son rôle lorsqu'il empêche, par son ingérence, le libre jeu des forces en n'importe quel domaine. Le domaine de l'économie est, conséquemment, un terrain clos où règne la ,spontanéité sociale". ${ }^{3}$

On trouve chez ces libéraux une foi aveugle en cette spontanéité. Les forces qui s'engagent sur le champ économique, n'ayant pas d'obstacles qui empêchent leur libre expansion, seront celles qui devront créer un ordre économique acceptable et celles qui, à la fin, déclancheront l'amélioration de la condition des classes salariées. ${ }^{4} \mathrm{~A}$ celles-ci, la démocratie leur offre, comme instrument de libération, la liberté d'association. ${ }^{5}$

1 Cf. n. 4, p. 9 de cet article.

${ }^{2}$ Emilio Castelar, El socialismo y la democracia, dans son journal La Democracia, 26 mai I 864 , no. 123 , p. I.

${ }^{3}$ Cf. l'entrefilet polémique de La Democracia, 22 mai 1864 , no. I 20 p. 2, où l'on accuse le socialisme d'„absolutista”, parce qu'il est porté pour "la negación de la propiedad natural [...]; la organización del trabajo por el Estado [...]; la tasa de los salarios [...]; los medios de evitar la libre concurrencia [...]”. „El colega [c.-d̀.-d., La Discusión] no confia en la espontaneidad social.” - Ceferino Tresserra, dans sa brochure ¿Los anarquistas, los socialistas y los comunistas, son demócratas?, o.c., pp. 73-74, exprime graphiquement l'idée libérale de l'État, en disant que des principes démocratiques „resulta un Estado insaboro, incoloro e inhedoro [sic!] en filosofia, en cuestiones sociales, garantizador sólo de los derechos y de las libertades de todos."

${ }^{4}$ Emilio Castelar, art. cit. n. 2, écrit: „El trabajador debe confiar en que la libertad majorará su condición social.” „El bienestar material de los pueblos [...] es un problema que depende $[\ldots]$ de muchas relaciones. ¿Cómo se resuelve? Dejando en libertad todas estas relaciones, para que por su propia virtud traigan el mejoramiento de las clases que padecen." „La democracia no puede ofrecer para resolverlo [le problème social] más que la libertad de pensamiento para que lo estudie; el sufragio universal para que arme de sus derechos al pueblo; la libertad de trabajo, la libertad de crédito, la libertad de cambio que ha de fundar grandes relaciones sociales; y sobre todo, la libertad de asociación."

5 Du même article: „La asociación añade fuerzas al obrero, le salva en las crisis económicas, le socorre en las enfermedades, le arma contra los intereses contrarios [...], fecundando la propiedad, producirá indudablemente mañana, cuando tenga toda su latitud, inmensos beneficios al trabajo." 
Ce schème d'affirmations, où l'on remarque la liberté, en tant que formule suprême, ainsi que l'existence de certaines „lois naturelles” 1 qui exigent que l'on écarte toutes sortes d'obstacles qui en empêchent la réalisation spontanée, permet à la fin l'anathématisation du socialisme. L'école socialiste nie la valeur suprême de la liberté et méconnaît la valabilité des "lois naturelles". ${ }^{2}$ Elle veut, pour tout dire, établir ses ,arrangements artificiels et arbitraires" dans la société. ${ }^{3}$

\section{Cette polémique éveilla l'intérêt des membres du parti dans tous les coins du pays. ${ }^{4}$}

La division des opinions subsista, à travers toutes sortes de changement, même après le célèbre manifeste du is mars I 865 , réprimé par la censure gouvernementale et publié loin de Madrid mais pas avant le mois de juillet de la même année. ${ }^{5}$ Ce document, de caractère clairement individualiste, ${ }^{6}$ bien que signé par toutes les personnalités du parti, hormis Pi y Margall qui s'était écarté de la politique, ${ }^{7}$ était destiné à mettre fin à toute hostilité à l'intérieur de la démocratie. Cependant, il n'en fut pas ainsi.

1 Du même article: „La sociedad es un ser real, objetivo, con propia vida, con leyes tan naturales e inevitables como las leyes de la mecánica celeste [...]. las leyes sociales no son en su fondo y en su forma, sino las mismas leyes de la naturaleza. La ley característica de la naturaleza humana $[. .$.$] es la libertad."$

2 J. Güell y Mercader, El socialismo es la reacción, dans La Democracia, 22 mai 1864 , no. I20 P. I., affirme du socialisme qu'il „es hoy una teoría [...] que, en más o menos escala, atenta al derecho humano"; qu'il est un „sistema reglamentario de la sociedad que atenta a las leyes naturales en que ésta vive y se desarrolla”; et il le décrit comme „el desconocimiento de las leyes naturales que rigen en todo."

${ }^{3}$ Emilio Castelar, El socialismo y la democracia, dans La Democracia, 26 mai I864, no. 123, p. I : „Nosotros [les individualistes] queremos la sociedad con sus leyes naturales y divinas; vosotros [les socialistes] la sociedad con vuestras combinaciones artificiales y arbitrarias."

4 Cf. les lettres publićes par La Democracia et par La Discusión à propos de la polémique au sujet du socialisme, dans les numéros de mai et juin I864. La Discusión du 30 mai, I, I I et 23 juin, publie des lettres d'ouvriers de Madrid, de Reus et de Valence qui demandent qu'on continue la polémique. - Anselmo Lorenzo, dans El proletariado militante, Barcelone 1902, pp. 19, 33 et 34 affirme que l'assimilation des concepts exposés dans la polémique lui fut d'une grande utilité, ainsi qu'à d'autres internationalistes de Madrid, pour se préparer à recevoir le message de Fanelli.

5 On peut le trouver dans le journal démocratique de Barcelone, El Comercio de Barcelona, 9 julliet $\mathrm{r} 865$, no. $36 \mathrm{I}$, pp. I et 2 ; et aussi dans le no. 49 I, 18 novembre 1865 .

B On y manifeste l'intention de „realizar el advenimiento del cuarto estado, del pueblo, al goce de los derechos políticos", mais on y remarque que, quoique „la democracia aspira a resolver el problema social [...] declara que nunca desconocerá ni mutilará los derechos inherentes a la personalidad humana". On y présente l'un de ces droits, la propriété, comme „tan natural, tan legítimo, tan fundamental como todos los demás derechos individuales." Cf. El Comercio de Barcelona, 9 julliet 1865 , no. $36 \mathrm{I}$, p. I.

7 E. Rodríguez Solís, Historia del partido republicano español, Madrid, v. II, I893, p. 562. Cf. aussi Francisco Caravaca, Pi y Margall, Barcelone I935, p. 63. 
C'est à Barcelone spécialement que la discorde devint publique à l'occasion de la nomination des Comités local et provincial du parti, dans une réunion qui eut lieu sur la Place des taureaux le s novembre I 865..$^{1}$ Une intervention de Ceferino Tresserra, soumettant au vote une proposition qui devrait éclaircir les positions, avec l'acceptation par tous ceux qui étaient présents du manifeste du is mars, provoqua un vrai vacarme entre le secteur individualiste et le secteur socialiste du parti. ${ }^{2}$ Malgré tout, on élit les comités prévus. Néanmoins, malgré les protestations de ces comités et de beaucoup d'autres éléments démocratiques, le vote se répéta quelques jours après ${ }^{3}$ et il parait que la nouvelle direction arriva à s'imposer. D'après l'organe du parti à Barcelone, ceux qui furent élus dans la réunion de la Place des taureaux sympathisaient avec le socialisme. ${ }^{4}$

Le mouvement ouvrier à Barcelone était bien loin d'être absent de ces dissensions idéologiques, et il était en rapport avec les fractions nées dans le parti démocratique à cause de ces dissensions-là.

\section{LE MOUVEMENT OUVRIER BARCELONAIS}

Quand on examine la réalité de tout près, on voit qu'il est peut-être exagéré d'employer le terme de mouvement au sujet des activités de la classe ouvrière, aux temps qui précédèrent la chute d'Isabelle II. II est vrai que le programme associationniste, présenté par ses organes

1 A Barcelone, l'organisation du parti démocratique se fit plus lentement qu'à Madrid. El Comercio de Barcelona du 4 décembre I 864, dans son article Otra circular révèle qu'à ce moment-là les comités et les réunions démocratiques en étaient encore au stade d'organisation. - Nous manquons aussi d'une étude de la structure du parti démocratique qui, pour être complète, devrait étudier à fond l'influence, le programme et l'origine des sociétés secrètes, qualifiées grosso modo de "Carbonarias", auxquelles appartenaient la plupart des militants démocratiques. - E. Rodríguez Solis, dans son livre Historia del partido republicano español, vol. II mentionné; et E. Vera y Gonzalez, Pi y Margall y la política contemporánea, Barcelone, vol. I, p. 4 I 2, nous apportent des données élémentaires à propos de l'existence de ces sociétés. Cette dernière référence nous décrit l'intervention des sociétés secrètes dans la révolution de $\mathrm{I} 82 \mathrm{I}$ à Barcelone. - Il est très important de lire, afin d'avoir une idée de tous les aspects qu'a ce problème en Europe, le livre d'Armando Saitta, Filippo Buonarroti, contributi alla storia della sua vita e del suo pensiero, 2 vols., Roma, I950 et $195 \mathrm{I}$. L'Article d'Arthur Lehning, Buonarroti and his international secret societies, dans International Review of Social History, d'Amsterdam, (1956) pp. I I 2-1 40, ne se limite pas à analyser le livre en question de Saitta, ainsi que deux autres de Galante Garrone qu'on mentionnera par la suite, mais nous indique aussi une piste à suivre pour ce qui touche aux problèmes de l'Espagne: le dossier des Archives Nationales de Paris, no. F. 7, 6684, qui est encore à exploiter.

2 Cf. El Comercio de Barcelona, 7 novembre 1865 , no. 480 , p. I.

${ }^{3}$ Les 19,20 et 21 novembre. Les résultats de ces votes dans El Comercio de Barcelona, 23 novembre 1865 , no. 496 , p. I. Les protestations ont été notées dans les actes du scrutin, publiées intrégralement par le même journal dans le numéro du 28 novembre 1865 , no. $50 \mathrm{r}, \mathrm{pp}$. $\mathrm{x}$ et 2 . Les noms de ceux qui protestèrent y sont notés.

4 Cf. El Comercio de Barcelona, I décembre I 86, no. 504, p. 2. 
de presse et proclamé par le Congrès Ouvrier de i 865 comme la solution des malheurs qui pesaient sur la classe ouvrière, faisait preuve d'une certaine conscience de classe, et formulait quelques buts à atteindre. Mais, d'autre part, la défense légale de s'associer qui pesait sur les ouvriers, ainsi que la crise qu'il y avait à l'Ateneo Catalán de la Clase Obrera - un instrument très important du mouvement au point de vue théorique - devaient enrayer notamment tout essai de donner quelque cohérence et quelque élan aux aspirations de la classe ouvrière.

\section{LA LOI ET L'ASSOCIATION}

Juan Uña Sarthou et Práxedes Zancada nous donnent, dans leur ouvrages respectifs Las asociaciones obreras en España et El obrero en España ${ }^{1}$ une esquisse rapide sur l'évolution des lois qui ont trait à l'association en Espagne. ${ }^{2}$ Depuis le décret des Cortès de Cadix, du 8 juin I 8 I 3 , dans lequel on déclarait les professions libres et les réglémentations corporatives périmées, jusqu'à la liberté d'association octroyée le 25 octobre i 868 par le Gouvernement provisoire issu de la révolution de septembre, les associations ouvrières ont évolué à travers une série d'alternatives de perte de prestige, de réhabilitation, de transformation en sociétés de secours mutuels et en coopératives. Les besoins de chaque période d'une part et le désir continuel de mettre en vigueur les idées libérales, abolutistes, coopérativistes, etc., ont marqué le rythme de cette évolution, qu'il faut encore étudier sérieusement non seulement dans son aspect national quand elle se réflète dans la législation du gouvernement de Madrid, mais plus spécialement dans son aspect local, tout en tenant compte de l'instabilité à laquelle elle fut soumise à Barcelone - où les sociétés ouvrières se sont notamment développées - par l'autorité civile et militaire de la province. ${ }^{3}$

Bien que, d'après la loi, il n'y eût pas de liberté d'association entre I 864 et I866, nous savons par la presse ouvrière, El Obrero et La Asociación, ${ }^{4}$ que, grâce au „,régime de tolérance établi par le général

1 Madrid I 900 et Barcelone 1902, pp. 296-300 et I 2 I-1 54 .

2 Le livre d'A. Marvaud, La question sociale en Espagne, Paris I91o, s'inspire, en bonne part, de ces ouvrages.

${ }^{3}$ A. Gusart, dans El Obrero, 25 septembre I 864, no. 4, p. 26, Tarifa y Jurado, taconte la dissolution des associations en Catalogne en I 856 (après deux ans de progressisme). Une étude consciencieuse de ce thème, devra mettre au clair la portée des mesures restrictives, les différentes pressions qui en furent l'origine, etc. Un essai très limité a été celui de Federico Urales, Reseña histórica del movimiento obrero español, dans La Revista Blanca, (Barcelone), le I juin 1926, pp. 22-26.

4 El Obrero, dirigé par Antonio Gusart Vila, fut publié la première fois à Barcelone, le 4 septembre I 864 et disparut dans le cours de 1866 , probablement après la révolution de juin de la même année. La date du 7 janvier I 866, donnée par J. Díaz del Moral, Historia 
Dulce et à la bienveillance du gouverneur civil", des associations ouvrières d'un caractère public agissaient en fait à Barcelone. ${ }^{1} \mathrm{Ce}$ régime de simple tolérance obligeait les associations ouvrières de vivre à la merci ,du caprice de n'importe quel mandarin". ${ }^{2}$ On comprend donc qu'il y avait constamment des pétitions afin de changer en régime légal ce régime de tolérance. ${ }^{3}$

Comme conséquence des insurrections du général Prim en janvier et juin 1866 , les réactions des autorités coupèrent court aux espoirs d'arriver à un régime de liberté légale pour les associations ouvrières et, avec la suppression de El Obrero et de La Asociación elle cachait dans l'obscurité la plus complète les vicissitudes du mouvement ouvrier barcelonais entre juillet I 866 et la révolution qui se déclencha deux ans après. ${ }^{4}$

de las agitaciones andaluzas, Madrid 1929, p. XXV, et par M. Garcia Venero, Historia de las Internacionales en España, Madrid 1956, p. 61, n. 2, comme celle de la disparition de El Obrero, est donc inexacte. La Asociación de 1o juin I 866, no. 10, p. Izo renvoie à un article publié dans El Obrero du I 3 mai 1866, no. 88, tout en polémisant contre lui. - La Asociación fut publiée aussi à Barcelone, pour la premic̀re fois le I avril $\mathrm{I} 866$, sous la direction de José Roca y Galés; on ne publia que quatorze numéros, jusqu'au 8 juillet I 866 . Tous ces numéros, sauf le numéro 2, du 8 avril, se trouvent à l'Institut International d'Histoire Sociale d'Amsterdam.

1 Cf. A. Gusart, Nuestro objeto, dans El Obrero, 4 septembre I864, no. I p. 2. - Dans El Obrero on trouve citées la société des tailleurs de pierres (Tarifa y jurado, 25 septembre I 864, no. 4, pp. 25-27); des tisseurs de voiles (article de Ramón Cartañá, ibid., pp. 27-28); des chaisiers (ibid.); des tisseurs de coton (entrefilet du no. 5, 2 octobre 1864, pp. 36-37); l'ancienne société des tisseurs (entrefilet du no. 60, 22 octobre I865, pp. 348-349); et la coopérative La Propagadora del Trabajo (no. 62, 5 novembre 1865, p. 376). - Et dans La Asociación la coopérative, déjà citée, La Propagadora del Trabajo (no. I, I avtil r866, p. 6; no. 3, Is avril I 866, pp. 37-38); on parla avec éloge de cette coopérative au cours du Congrès Ouvrier de Barcelone, décembre r 86 s (cf. le journal El Telégrafo, de Barcelone, 25 décembre 1865 , no. 360, p. 8790). Une autre coopérative est mentionnée aussi dans La Asociación: celle des briquetiers de Tarrasa (no. 5, 6 mai i 866, pp. 62-63).

2 Cf. Las asociaciones y la ley de asociación, dans La Asociación, I avtil i 866, no. 3, p. 35.

3 Cf. la lettre adressée au Président du Conseil des Ministres après le Congrès Ouvrier de décembre 1865 , dans laquelle on réclame la liberté d'association, dans El Obrero, 7 janvier 1866, no. 7I, Pp. 5I 8-5I9. - Dans La Asociación, 22 avril I 866, no. 4, Pp. 46-47, on écrit: „Si el gobierno tolera las asociaciones, barrena la ley; si no las tolera, cada día se descubrirán sociedades secretas y se dará motivo para que se hable y se comente sobre soñadas conspiraciones [...]". On conclut avec cette affirmation de Lammenais: „¿ Queréis acabar con las sociedades secretas? Estableced una pública". - On trouve aussi des allusions à des sociétés ouvrières secrètes dans A. Gusart, Nuestro objeto, El Obrero, 4 septembre I 864, no. I p. 2 ; La asociación productiva, ibid., 25 décembre 1864 , no. 17, p. 130. Cf. aussi l'article de Federico Urales, cité dans le n. 3, p. I I.

4 Dans les déclarations de différents ouvriers à l'occasion du Congrès Ouvrier de Barcelone, en juin 1870 , on trouve des données à propos de la vie associée des ouvriers. Elles sont pourtant très sommaires et elles ne font que confirmer l'impression qu'on a de la décadence du mouvement ouvrier immédiatement avant la révolution de $\mathrm{s} 868$. Cf. les déclarations de Fornells, La Federación, 23 julliet I 870 , supplément no. 3, p. 5; de Ramón Solá, ibid., p. 6 et supplément no. 4, p. 7; d'Antonio Illa, ibid., supplément no. 4, p. 7; de Joaquín 


\section{L',ATENEO CATALÁN DE LA CLASE OBRERA”}

Bien que la vie des associations ouvrières se développât en des conditions précaires, faute de garanties légales qui pourraient donner quelque stabilité à leur existence, il y avait une organisation culturelle, l'Ateneo Catalán de la Clase Obrera, qui par sa nature même était appelée à jouer un rôle important dans le mouvement ouvrier mais à l'intérieur de laquelle il y avait des obstacles qui l'empêchaient d'atteindre ce but.

Max Nettlau s'est probablement trop pressé quand il a comparé sans observer aucune nuance l'Ateneo Catalán de la Clase Obrera (fondé, d'après ses références, en I 86I), au „Fomento de las Artes" de Madrid. ${ }^{1}$ Il est vrai que tous les deux accueillirent ces ouvriers qui, plus tard, devaient figurer comme des membres détachés de l'Internationale. Mais à en juger d'après les nouvelles publiées par El Obrero, ainsi que par les références d'Anselmo Lorenzo, le développement du Fomento de las Artes fut bien plus cohérent que celui de l'Ateneo. ${ }^{2}$ Il nous reste une brochure éditée à l'occasion de certaines dissensions intérieures vers la fin de l'année 1864, ainsi que des nouvelles que nous donnent, au sujet de l'Ateneo, les publications El Obrero et La Asociación, pour nous faire connaitre la vie de cette institution avant I 868.

Le malaise intérieur eut son origine dans la décision prise par la direction de donner une plus grande étendue à la section récréative de l'Ateneo. Cette décision, d'après les quarante associés qui protestèrent, „entacha une corporation, dont le but principal a été et devait être toujours l'éducation de l'ouvrier". ${ }^{3}$

L'intervention de la première autorité de la province, jugeant l'affaire contre le Conseil de l'Ateneo, remit, pour l'instant, les affaires

Riera, ibidem; de Pablo Sampere, ibid., supplément no. 4, p. 8, et de José Rubau Donadeu, ibid., supplément no. 5, p. 9 .

${ }^{1}$ M. Nettlau, Zur Geschichte der spanischen Internationale und Landesföderation, dans Archiv für die Geschichte des Sozialismus und der Arbeiterbewegung, I929, pp. I I et I 2. ${ }^{2}$ Sur le „Fomento de las Artes" de Madrid, cf. El Obrero, no. 8, 23 octobre i864, pp. 6I-63; no. II, I3 novembre I 864, pp. 85-86; no. I 2 , 20 novembre I 864, pp. 92-94; no. I3, 27 novembre I 864 , p. I04; no. I4, 4 décembre I 864 , p. II I; no. I 5 , II décembre I 864 , p. I 20 ; no. I6, I 8 décembre 1864 , p. I 28; no. 17.25 décembre 1864 , Pp. I 34-1 35 ; no. 18 , I janvier I 865, pp. I40-I 4I et no. 21,22 janvier 1 865, p. I62. - Cf. aussi Anselmo Lorenzo, El proletariado militante, v. I, Barcelone 1902, pp. 17 ss.

3 Aclaración de hechos que varios socios del Ateneo Catalán de la clase obrera dirigen a todos sus consocios y compañeros, Barcelone, le 20 janvier I865, p. 4. Parmi les signataires on trouve Rafael Farga Pellicer et d'autres, tels que Jaime Balasch, Jacinto Pagés et Jaime Boguñá, qui participèrent au Congrès Ouvrier de 1870 et y jouèrent un rôle remarquable. Leurs activités ont eu beaucoup d'importance pour l'histoire de la Première Internationale. 
en place. ${ }^{1}$ Cependant, les désaccords entre le Conseil et les associés ne s'arrêtèrent pas là ${ }^{2}$, car il semble bien que ces désaccords touchaient à des questions fondamentales. Certaines insinuations imprimées dans El Obrero font supposer que la direction suivait une ligne politique qui sympathisait avec le progressisme. ${ }^{3} \mathrm{La}$ documentation est insuffisante pour nous permettre de voir précisément où prenaient corps ces sympathies politiques. Antonio Gusart en vient à dire, dans un des articles de El Obrero, sans détailler plus, que l'orientation suivie par l'Ateneo contribuait à désunir les ouvriers et à étouffer l'esprit d'association, si enraciné dans la classe ouvrière du pays. ${ }^{4} \mathrm{~L}$ 'accusation, prise littéralement, était véritablement grave: l'esprit d'association était un des distinctifs les plus chers au mouvement ouvrier barcelonais.

\section{LA PRESSE OUVRIÈRE ET LES IDÉES ASSOCIATIONNISTES}

Antonio Gusart Vila, directeur d' El Obrero, fut un personnage dont les caractéristiques individuelles nous sont, jusqu'à présent, tout à

1 Ibid. pp. I4 et I s, et El Obrero, no. 21,22 janvier I 865, p. I63.

2 Cf. El Obrero, no. 28, I 2 mars 1865, Pp. 218-219; no. 26, 26 février I865, pp. 201-202; et no. 27, 5 mars I 865, pp. $213-214$.

${ }^{3}$ El Obrero du 23 juillet I 865, no. 47, pp. 139-142 réfute les accusations d'un article de La Corona (journal progressiste barcelonais) qui loue le procédé du Conseil. Un autre article d' El Obrero, du 30 juillet de la même année, no. 48, p. I 5 I, fait allusion au sujet des problèmes de l'Ateneo, „à celui qui, derrière la protection du titre „riche”, veut changer l'honorable et laborieux fils du travail en un outil pour réaliser ses plans usurpateurs". - Nous avons un indice assez clair de l'influence de l'élément progressiste à l'Ateneo Catalán de la Clase Obrera, dans la lettre que le Président provisoire de la commission de Fomento de cette corporation, J. Espalteá, envoya le 20 janvier I 864 à D. Agustín Aymar, dirigeant progressiste barcelonais. Il lui faisait savoir que lui et D. Manuel Torrens Ramalló, également dirigeant progressiste, avaient été chargés de vérifier les comptes de l'A teneo. Il existe une lettre analogue, celle-ci signée par le Président du Conseil du Gouvernement, Antonio Doménech, du 9 janvier I 869 . Ces lettres se trouvent parmi les papiers d'Aymar aux Archives de la Bibliothèque Centrale de Barcelone, Ms. I 26o. - Finalement, il y a encore deux autres données pour appuyer cette supposition. L'une d'elles pourrait bien être une source d'information magnifique si on arrivait à trouver les archives personnelles de D. Pascual Madoz. Il s'agît d'une lettre de celui-ci à Víctor Balaguer, du 29 Octobre I 862, dans laquelle l'expéditeur demandait au destinataire à propos de l'Ateneo: „Pourriez-vous me dire, quand vous aurez un moment de détente, ce qu'est l'Ateneo Catalán de la Clase Obrera, quelle est sa pensée politique, quelies idées économiques il professe, et quelles aspirations sociales il nourrit? Je suis intéressé, avec une certaine réserve, à connaître votre opinion, que je n'attends également qu'avec réserve de votre part." (On trouve cette lettre aux Archives de la Bibliothèque-Musće Balaguer de Villanueva y Geltrú, no. 472, et elle est publiée par R. Olivar Bertrand: Así cayo Isabel II, Barcelone I955, p. 323, appendice documentaire no. 36.) - L'autre donnée est une lettre de Prim à Balaguer, du 21 septembre I863, dans laquelle celui-là accuse réception du "titre et réglémentation de l'Ateneo de Obreros". (Aux mêmes Archives, no. 357. Publiée par R. Olivar Bertrand: El caballero Prim, Barcelone, vol. II, I952, p. 438.)

4 Cf. A. Gusart, La oposición, dans El Obrero, 2 mars 186, no. 28, p. 219. 
fait inconnues. Par contre, les quelques données que nous avons à propos de son orientation politique, suffisent à nous faire comprendre le contexte où il faut placer ses idées associationnistes, répandues par son organe de presse.

Comme conséquence de la polémique au sujet du socialisme entre Pi y Margall et Emilio Castelar, de nombreux démocrates de Barcelone, parmi lesquels Antonio Gusart, Jaime Tubau, Pablo Alsina, Francisco Suñer Capdevila et les frères Julio et José Rubau Donadeu, publièrent ensemble, le ro juin 1864 , un „Manifiesto a los demócratas españoles", où ils déclarèrent ouvertement leurs sympathies pour le socialisme, qui fait passer sur le terrain des faits la liberté, proclamée seulement en droit par les démocrates (individualistes). „Nous ne pouvons pas accepter", avouent-ils - ce qui semble pouvoir être une phrase explicative de l'assertion antérieure -, „que l'Etat n'ait pas lui-même le devoir indispensable d'écarter tous les obstacles qui s'opposent à la réalisation de la justice dans tous les stades". ${ }^{1}$

Malgré tout, leur socialisme n'a pas sont point de départ dans des convictions théoriques définitivement acquises. Ils considèrent que la polémique doit suivre pour que la lumière devienne plus claire, parce que le thème n'a pas encore été résolu. Et ils se contentent de cette formule empirique: „L'action collective commence là où l'initiative individuelle n'est pas possible".

L'orientation démocratique-socialiste de Gusart, avec toutes les atténuations mentionnées, est confirmée, non seulement par son opposition générale aux consignes du Conseil de l'Ateneo Catalán de la Clase Obrera, mais aussi par le fait d'avoir été parmi les élus du Comité provincial du parti, à Barcelone, à la réunion de la Place des taureaux, le 5 novembre 1865 .

Cependant, son socialisme ne se montre qu'à petite échelle dans son activité de journaliste. Dans toute la collection de la revue, il se trouve, d'une part, seulement une légère allusion à l'intervention désirable „d'un pouvoir supérieur et impartial” (l'Etat?) dans les conflits entre le capital et le travail, lorsque la situation se trouve dans une impasse ; ${ }^{2}$ et, d'autre part, une phrase qui, dans l'ambiance polémique que nous avons tracée, a toute la valeur d'une accusation. Les démocrates individualistes prétendaient, comme nous l'avons dit, que les malheurs des classes laborieuses se résoudraient de façon spontanée si l'on laissait les forces économiques agir librement. Gusart affirme que „le caractère d'épouvantail" du mot socialisme est dû au

1 On peut voir ce Manifeste dans le journal La Discusión 21 juin I864, no. 2606, pp. I et 2.

2 Cf. A. Gusart, El proletariado, dans El Obrero, 18 septembre I 864, no. 3, p. I 8. 
„nombre de ceux qui ont de l'intérêt à ce qu'il y ait toujours des quantités de malheurs". ${ }^{1}$

On doit peut-être chercher les raisons de cette sobriété dans l'ambiance de convictions solides et, sûrement, dans le caractère tout à fait apolitique de l'hebdomadaire El Obrero.

La revue de Gusart a un programme nettement associationniste. Déjà dans son premier numéro se manifeste nettement la conviction que l'association est destinée à apporter de grands avantages à la classe ouvrière. L'association libre, „doit contribuer à harmoniser et à refondre les diverses classes de la société". ${ }^{2}$

Le programme d'association poussé par El Obrero prétendait, en effet, apporter une solution au problème des relations entre le capital et le travail. Le premier avantage de l'association était de donner à l'ensemble des ouvriers réunis dans une collectivité, une force que, en tant qu'individus, ils n'avaient pas. Cette nouvelle force venait constituer un élément d'équilibre dans un monde où la prééminence absorbante de la force du capital rend impossible la régénération du prolétariat. $^{3}$

Il faut pourtant noter qu'il ne s'agissait pas d'une force ouvrière collective organisée ou organisable pour déclencher une lutte violente contre le capital. ${ }^{4}$ La résistance au capital, la simple coalition conçue pour se procurer certains fonds afin de soutenir les grèves qui, à un moment donné, pourraient faire plier la force des patrons, est condamnée sans ambages. ${ }^{5}$ "Si le capital employé pendant des périodes de

1 Voici le paragraphe entier: „La palabra socialismo no es para nosotros más que una palabra que demuestra hasta la evidencia un sinnúmero de deformidades en el organismo social; y como hay muchos interesados en mantener tanta exhuberancia de males, de aquí el haber dado a esta palabra el carácter espantador que hoy tiene". A. Gusart, La mendicidad y las teorías sociales, dans El Obrero, 4 juin 1865 , no. 40, p. 5r. - En outre, à l'occasion de la mort de Jaime Tubau, A. Gusart loue les idées démocratico-socialistes. El Obreto, I9 mars 1865 , no. 29, p. 225.

2 El Obrero, no. I, 4 septembre I 864, p. 2.

3 Cf. tout l'article d'A. Gusart, El orden y las sociedades obreras, dans El Obrero, 23 avril I 865 , no. 34, pp. $265-266$. „La asociación”, écrit-il à la p. 266 , „regula la potencia absorbente del capital y con la fuerza de la colectividad regenera al hombre."

4 Ibid.

5 Cf. A. Gusart, La asociación productiva, dans El Obrero, 25 décembre 1864, no. 17 , p. 1 30: „Nuestra misión es de paz [...]; venimos a operar una revolución en la marcha de las asociaciones obreras [auparavant elles étaient presque exclusivement sociétés de résistance], revolución que cambiará de una manera progresiva las condiciones de esa lucha empeñada entre el capital y el trabajo, y evitará gastos cuantiosos a entrambos contendientes [...]. Ese cambio se operará [...] sin costar una lágrima, sin conducir a ningún obrero a la desesperación, ni a la miseria; porque, modificándose las condiciones de la lucha, no será preciso resistir de la manera ostensible que se ha hecho hasta la actualidad". Cf. A. Gusart, Cuatro palabras a nuestros adversarios, dans El Obrero, 27 novembre 1864, no. 1 3, pp. 97 et 98 . 
résistance afin de conserver le salaire [...] avait été destiné à la production, combien autre ne serait la condition de l'ouvrier!" ${ }^{1}$ On disait simplement que la juste répartition des salaires résulterait spontanément de l'équilibre des deux forces, le capital et le travail, à condition que celles-ci fussent mises dans des conditions de partis égaux. ${ }^{2}$ Cette vague formule veut signifier, sur le plan concret, que les deux partis doivent tomber d'accord au sujet de certaines taxes et qu'ils doivent créer des commissions mixtes pour résoudre en justice les cas en litige. ${ }^{3}$

Mais les bienfaits de l'association ne s'arrêtent pas là. Le but logique vers lequel tend la „force collective" des ouvriers groupés, c'est la coopérative. ${ }^{4}$

Bien que la coopérative soit présentée, avec la plus grande franchise, comme un idéal, dans le journal dirigé par Gusart il y manque quelque exposé plus complet du système coopératif, de son histoire et de ses avantages. Il n'y a pas de raisons de croire que cette absence soit une conséquence de quelques causes cachées, vu qu'au Congrès Ouvrier de I 865 , convoqué par l'initiative de Gusart les 24,25 et 26 décembre de la même année, ${ }^{5}$ le thème de la coopérative était inclus dans le programme, et ce thème a constitué, en fait, un des points les plus importants.

Le propos de Gusart était de propager, par ce Congrès Ouvrier, dans l'ordre doctrinal, les avantages de l'association en général et tout particulièrement du système coopératif; ${ }^{6}$ dans l'ordre pratique, il cherchait à donner une orientation cohérente aux organisations ouvrières existantes, ainsi qu'à pousser la création d'associations ou-

1 A. Gusart, La asociación productiva, dans El Obrero, 25 décembre 1864 , no. 17, p. I30.

2 A. Gusart, El orden y las sociedades obreras, dans El Obrero, 23 avril I 865, no. 34, p. 266.

${ }^{3}$ Cf. A. Gusart, Tarifa y Jurado, dans El Obrero, 25 septembre 1864 , no. 4, p. 26. Cf., du même auteur, Cuatro palabras a nuestros adversarios, ibid., 27 novembre 1864 , no. 13 , p. 98 ; et l'article anonyme La situación en Reus, ibid. p. roo.

4 A. Gusart, La asociación productiva, dans El Obrero, 25 décembre 1864, no. 17, pp. 129-13I.

5 A. Gusart, Un congreso de obreros, dans El Obrero, 3 septembre 1865, no. 53, pp. 229-23 I, publié également dans le no. 67, xo décembre $x 865$, pp. 454-455.

6 Voici les sujets de ce Congrès:

"I ${ }^{\circ}$. - ¿Puede la asociación redimir al proletariado, devolviendo al obrero la libertad de acción que le falta?. - En caso afirmativo, ¿cuál es la marcha que deben seguir las sociedades para evitar en lo posible el malestar de la clase obrera, ya que hasta la actualidad no han mejorado su situación?

$2^{\circ}$. - Las sociedades cooperativas, ¿qué ventajas reportan a los asociados y qué probabilidades tienen de buen éxito?

$3^{\circ}$. - $\Varangle$ Seria conveniente establecer una federación para el mejor éxito en las operaciones y que sirviera de base a la marcha progresiva de las sociedades?". Ibid. 
vrières là où elles n'existaient pas encore, tout en propageant les coopératives. ${ }^{1}$

Les renseignements à propos de ce Congrès auquel ont participé quarante sociétés ouvrières, ${ }^{2}$ sont très limités. D'après l'information de la presse, on constate que le Congrès se prononça pour la liberté d'association, pour le principe de coopération et pour la fédération des sociétés ouvrières, ${ }^{3}$ tout en respectant leur autonomie. ${ }^{4}$ En plus, on sait que parmi les assistants prédomina la tendance à exclure l'intervention de l'Etat dans le domaine de l'économie. ${ }^{5}$ Les journaux libéraux essayèrent d'attribuer à ce fait une portée politique: les ouvriers, d'après eux, se déclarèrent ennemis du socialisme. ${ }^{6} \mathrm{El}$ Obrero se borna à protester contre cette interprétation, mais il s'abstint de nier ce fait. ${ }^{?}$

Le Congrès décida, en plus, de faire parvenir à la Présidence du Conseil des Ministres une lettre pour demander la liberté d'association. ${ }^{8}$

Le journal de José Roca Galés, La Asociación, défendait cette même pensée associationniste, bien que l'idée coopérativiste y soit exprimée avec plus de force, et de consistence, dans une langue dont le style et la grammaire sont bien plus clairs, et bien que l'inspiration politique soit très différente.

L'orientation politique de Roca Galés se montre également lors de la polémique de r 864 au sujet du socialisme. Dans une lettre adressée à Castelar, le 3 I mai 1864 , il se déclare partisan des idées des démocrates individualistes qui s'opposent ouvertement à toute intervention de l'Etat sur le terrain de l'économie. ${ }^{9}$

1 Ibid., p. 230.

2 El Obrero, 3 I décembre I 865 , no. 70, p. 504.

3 Il s'agit peut-être de reprendre la ligne de conduite de la fédération des sociétés attachées à la branche du coton, qui, en I 854 , avait institué l',Unión de Clases”, société qui, d'après les références de Juan Salas Antón, publiées par S. Magalhaes Lima, en O Socialismo na Europa, Lisbonne 1892 , p. 3 I 5 , était au commencement bien puissante, mais qui s'affaiblit après de plus en plus jusqu'à sa disparition en 1868 .

4 La deuxième conclusion du Congrès est celle-ci: „Dicha unión [la fédération des sociétés ouvrières] no perjudicará en lo más mínimo la autonomía social de las mismas, pues cada sociedad girará dentro de la órbita que ella misma se trace." Cf. El Obrero, 3 I décembre 1865 , no. 7 o, p. 504 .

5 Le journal El Telégrafo de 26 décembre 1865 , no. 360, p. 8790, affirmait que „[...] salvo muy contadas excepciones, la tendencia general durante la sesión se inclinó a resolver la cuestión llamada social por medidas eminentemente liberales, rechazando toda intervención del Estado."

${ }^{6}$ Cf. ibid.; et, en outre, El Comercio de Barcelona, 28 décembre I 865, no. 70, p. 503.

7 El Obrero, 3 I décembre 1865, no. 70, p. 503.

8 Voir la lettre publiée dans El Obrero, 7 janvier 1866 , no. 71 , pp. 9 18- 519.

9 Cette lettre a été publiée dans le journal La Democracia, 3 juin I 864, no. 129, p. 2. On y lit, par exemple: „Nosotros somos demócratas; nada queremos del Estado sino lo que 
Voilà la tendance de La Asociación. Les problèmes concernant les difficultés entre le travail et le capital doivent être posés dans un domaine exclusivement économique, et indépendant de celui de la politique. On pourrait résumer par ces quelques mots le programme idéologique de la publication.

Cette formule renferme deux présuppositions. D'abord elle accepte comme un fait l'existence de ce qu'on désigne comme des "lois économiques", une sorte de normes naturelles qui doivent être à la base de l'activité économique. Ceci avec une confiance absolue qui ne manque pas d'un certain rationalisme. Dans la ligne de ces "lois naturelles", le coopérativisme permet de régler sans violence les relations entre le capital et le travail, ${ }^{1}$ et en exclut n'importe quelle intervention extra-économique. ${ }^{2}$

$\mathrm{La}$ deuxième présupposition est l'affirmation que l'Etat, dans le domaine politique, doit se limiter à assurer le climat de liberté qui permet la règne des "lois économiques" mentionnées. ${ }^{3}$

En somme, la fraction de la classe ouvrière barcelonaise qui se manifestait avec les caractéristiques d'un mouvement, se trouvait engagée suivant son programme dans les chemins de l'associationnisme coopératif, encouragée ouvertement par le courant démocratique individualiste, parce qu'elle se trouvait être d'accord avec le dogme fondamental des démocrates individualistes concernant la libre initiative et la libre concurrence en tant que lois naturelles du monde économique.

naturalmente nos pertenece: los derechos [...]. Por eso queremos primero libertad, y luego, como consecuencia de ella, el mejoramiento moral y material de la clase obrera. Para lograr su objeto, creemos que lo mejor es la absoluta libertad de asociación."

${ }^{1} \mathrm{La}$ déclaration d'intentions dans le premier numéro, est textuellement comme suit: „Queremos ver regulados ambos elementos de la riqueza pública [le capital et le travail] por leyes puramente económicas, sin que jamás puedan tratar de equilibrarse por la violencia. Porque las sociedades cooperativas tienden a este noble objeto, por ello lo son [sic] de nuestros más ardientes cariños." La Asociación, I avril I 866, no. r, p. 2. Dans ce même article, on dit des coopératives qu'elles sont „la última forma de la expresión científica de la asociación obrera." - Roca y Galés, dans son article De las asociaciones cooperativas comparadas con las de socorros mutuos y demás asociaciones obreras de Cataluña, dans La Asociación, 22 avril 1866 , no. 4, pp. 54-55, se prononce pour la solution coopérative, vu la valeur intrinsèque de cette idée même et parce que cette idée peut s'imposer sans violence, sans "necesidad de perturbar en lo más mínimo el orden social."

2 "La asociación libre, autónoma, no intervenida, ha de resolver los grandes problemas sociales". De la déclaration des intentions, La Asociación, I avril i 866, no. I, p.2.

3 "Las intervenciones sociales" - c.-̀̀-d., les interventions de l'État, cf. no. 6, I 3 mai I 866, p. 7 I - „han de ser cada vez menores, y el día en que la asociación individual esté sólo limitada por el deber, ése será el en que [sic] la asociación alcance sus mayores beneficios" (ibid. p. 2). - L'article El proletariado y el pauperismo, signé par UN LIBERAL, manifeste sympathie pour Louis Blanc, mais il lui reproche que l'État soit l'organisateur du travail. Cf. La Asociación, 3 juin 1866 , no. 9 p. 101. 
Cette même orientation coopérativiste se trouvait favorisée en même temps par le courant socialiste, sans que cela signifie, pour ceux qui en sont les partisans, que la formule "coopérative" épuise toutes les possibilités de résoudre le conflit qu'il y a entre le travail et le capital et de trouver la solution des problèmes de l'ensemble économique. Là, où prévalait la tendance socialiste, on mettait de l'obstination à ne pas créer de divorce entre l'Etat et les problèmes économiques, et à présenter le problème politique, pas simplement comme un problème de forme de gouvernement, mais comme une question très étroitement liée à la structure et au fonctionnement de l'économie du pays.

Tous les indices semblent renforcer limpression que le courant individualiste fut celui qui avait le plus d'influence sur ce que nous nous accordons à appeler le mouvement ouvrier antérieur à la chute d'Isabelle II. Le témoignage de Roca Galés qui, dans sa lettre à Castelar déjà mentionnée, déclare qu'en Catalogne les socialistes forment une petite minorité, ${ }^{1}$ peut sembler - et il l'est, en effet - un témoignage intéressé. Cependant, l'orientation prise par le Congrès Ouvrier de 186 , et qui s'oppose à l'intervention de l'Etat, la sobriété de l'organe de Gusart lui-même dans la manifestation de ses convictions les plus intimes, ainsi que les affirmations d'une lettre de Rafael Farga Pellicer adressée à Bakounine en août i 869 , époque d'une pleine liberté, corroborent l'affirmation de Roca Galés. D'après Farga Pellicer, il fallait même à ce moment - là propager „prudemment” le socialisme, afin de ne pas provoquer de scissions parmi la classe ouvrière; le socialisme n'était pas "aussi développé qu'on l'aurait désiré", et la grande majorité des ouvriers n'était considérée que comme "susceptible" d'être socialiste. ${ }^{2}$

Tout essai de traduire ces impressions en des chiffres concrètes qui expriment la portée et la profondeur de l'influence de ces deux courants doit rester vain puisqu'on ne dispose pas de données élémentaires qui puissent fournir une suffisante garantie scientifique. Il en est ainsi, par

1 „Muchos creen”, écrit Roca y Galés, „que, porque en Cataluña hay el gran cariño a la asociación, que ya todos somos socialistas. Es un error. $Y$ no negaremos que haya algunos que lo son; pero muy pocos que lo son por escuela y principio; otros, y éstos son los más, que aclarada la cuestión dicen que son socialistas porque son amantes de la asociación y sus derechos." Cf. La Democracia, 3 juin 1864 , no. I 29, p. 2.

2 "Aquí el socialismo no está tan desarrollado como fuera de desear; así es que el Centro Federal [des Sociétés Ouvrières, constitué en 1868 ] no ha decidido nada clara y terminantemente respecto a este punto tan interesante." "No obstante, he de participaros con placer que la gran mayoria de los obreros son susceptibles de ser decididamente socialistas.” „Vos, querido amigo y correligionario, comprendéis con cuánto cuidado y con cuánta prudencia ha de hacerse esta importante propaganda, para evitar futuras escisiones que retardarian más el triunfo de nuestra causa." La lettre est publiée par M. Nettlau, dans Miguel Bakunin, la Internacional y la Alianza en España, Buenos Aires 1925, pp. 45-47. 
exemple, du nombre de la population ouvrière ${ }^{1}$, d'associations ouvrières qui existent à cette époque et du nombre de membres-ouvriers groupés dans chacune d'elles. ${ }^{2}$ En tout cas, les précisions apportées dans les pages qui précèdent, peuvent faciliter la compréhension de l'état dans lequel se trouvaient les ouvriers barcelonais qui reçurent au commencement de 1869 le message de Fanelli, et de l'influence définitive exercée sur l'orientation du mouvement ouvrier de Barcelone, après la révolution du mois de septembre i 868, par le contact que Farga et Sentiñón eurent avec Bakounine au Congrès de l'Internationale à Bâle, en septembre 1869 .

1 Fernando Garrido, dans son ouvrage Historia de las clases trabajadoras, Madrid 1870 , p. 749 , nous donne une statistique des ouvriers industriels d'Espagne en I86r; et à la p. 767 une autre de la population agricole. Il s'agit de nombres d'une valeur scientifique imprécise, et qui ne sont donc pas très utiles. Selon le rapport de Farga y Pellicer au Congrès de l'Internationale à Bâle (septembre 1869), le nombre des travailleurs de toutes les professions, associés en Catalogne après I 868, période de liberté d'association totale, était $7.08 \mathrm{I}$ (cf. ce rapport dans O. Testut, Le Livre bleu de l'Internationale, Paris $187 \mathrm{I}$, pp. 135-140, et dans La Federación, 2 janvier I 870 , no. 23, p. 3). Les seuls ouvriers de la laine et du coton, localisés pour la plupart dans la Catalogne, étaient, selon Garrido, au nombre de 78.044. On peut soupçonner, d'après ces données, que le nombre des travailleurs associés était d'une importance relative, puisqu'il n'atteignait pas $10 \%$ du nombre d'ouvriers, après 1868 . On s'imagine la petite importance numérique des associés à une époque de simple tolérance ou de véritables persécutions.

2 Les statistiques publiées (p. e., Censo de la población de España, du 21 mai 1857 , Madrid; Resultados generales del censo de la población de España, le 31 décembre i 877 , Madrid I 879; l'étude de García Barzanallana, La población de España, Madrid i 872; ct plus spécialement encore celle de G. Colomer Codina, Movimiento de la población de Barcelona en el veintenio I 861 - I 880 , Barcelone 1883 ), ne nous fournissent que de données démographiques - naissances, décès, mariages, etc. - mais elles ne spécifient pas des détails de professions. D'autre part, à Barcelone, les registres de recensement furent détruits pendant la révolution de I936, d'après les informations de M. José Brú Jardi, Chef du Bureau Administratif de l'Institut Municipal de Statistiques de la Commune de Barcelone. Pour compléter l'ensemble, nous manquons encore d'études sur le niveau de vie, sur les salaires, sur la crise qui paralysa en I 864 l'industrie cotonnière catalane (crise due au manque de matières premières qu'on importait des Etats Unis, le transport en étant suspendu à cause de la guerre intérieure du pays exportateur). 